\title{
Grain rotation in nanocrystalline layers under influence of swift heavy ions
}

\author{
Ivo Zizak* \\ Berliner Elektronenspeicherring-Gesellschaft für Synchrotronstrahlung mbH, Albert-Einstein-Str. 15, 12489 Berlin, Germany \\ Nora Darowski, Siegfried Klaumünzer, and Gerhard Schumacher \\ Hahn-Meitner-Institute, Glienicker Straße 100, 14109 Berlin, Germany \\ Jürgen W. Gerlach \\ Leibniz-Institut für Oberflächenmodifizierung, Permoserstr. 15, D-04318 Leipzig, Germany \\ Walter Assmann \\ Ludwig-Maximilians-Universität München, Am Coulombwall 1, D-85748 Garching, Germany
}

\begin{abstract}
Significant changes in texture occur in nanocrystalline $\mathrm{Ti}$, TiN, and $\mathrm{NiO}$ layers during irradiation with $350 \mathrm{MeV}$ Au ions. The angle between ion beam and layer normal $\Theta$ was between $30^{\circ}$ and $70^{\circ}$. The major effect is a collective rotation of the nanocrystals. In case of $\omega$ layers the texture rotated by more than $70^{\circ}$ at an ion fluence $\Phi t=3 \times 10^{15} \mathrm{Au} / \mathrm{cm}^{2}$. In addition to grain rotation, the layers exhibit a shear motion like that observed previously with amorphous materials. Below about $1 \times 10^{15} \mathrm{Au} / \mathrm{cm}^{2}$ the grain growth is small and grain rotation is reversible, i.e. reversing the sign of $\Theta$ and applying the same $\Phi t$, the grains roll back into their original orientation. The second observed effect is the alignment of the grains, whose coalescence eventually leads to a mosaic crystal. However, grain rotation is absent or immeasurably small in micro-crystalline titanium. An attempt to understand the processes in nanocrystalline materials in terms of amorphous grain boundaries, as well as the disclination dipole diffusion along the grain boundary was made.
\end{abstract}

Key words: SHIM, Material Modification, Texture, Ion Hammering, Nanocrystalline Materials PACS: 61.80.Jh, 61.46.Hk, 68.35.-p

\section{Introduction}

About 20 years ago polycrystalline metals with average grain sizes $d_{g}<100 \mathrm{~nm}$ were first synthesized in bulk form (for a recent review see [1]). Since then the structure and mechanical properties of these materials have been of great interest. Based on the Hall-Petch relation, $\sigma_{Y}=a+b \cdot d_{g}^{-1 / 2}$ with $a$ and $b$ being constants, improved hardness and yield strength $\sigma_{Y}$ were expected with decreasing $d_{g}$. The limitation of this relation is found at grain sizes of typically 20 to $30 \mathrm{~nm}$, where sometimes a crossover from "normal" to "inverse" Hall-Petch behavior occurs (for a recent review see [2]). It is believed that this crossover results from a change in deformation mechanism: from one based on dislocation processes for $d_{g}>100 \mathrm{~nm}$ to one mediated by

\section{*}

Email address: ivo.zizak@bessy.de (Ivo Zizak). grain boundary processes for $d_{g}<30 \mathrm{~nm}$. This crossover could be reproduced by molecular dynamics simulations of nanocrystalline materials with random grain orientations $[2,3]$. According to these simulations a wide variety of grain boundaries and grain boundary structures exists in coarse-grained materials, whereas in nanocrystalline materials high-energy grain boundaries prevail, and they are amorphous $[2,3]$. While this point of view is experimentally corroborated for several ceramics (for a recent overview see [4]), it is not undisputed for metals and silicon because i) amorphous grain boundaries in metals have never been observed by high-resolution electron microscopy [4,5], and the structure of a grain boundary may depend on the way how the nanocrystals are constructed in the simulation [6], and ii) by extending molecular dynamics simulations of nanocrystalline silicon to time-scales of $10 \mathrm{~ns}$, it has been argued that amorphous grain boundaries relax into strongly distorted but ordered ones [7]. 
In this Paper we present experimental evidence for the collective rotation of nanocrystals induced by high-energy ion irradiation. This effect is absent or immeasurably small in micro-crystals. Obviously, the dynamics of grain boundaries in nanocrystalline materials play a crucial role in generating this rotation.

Swift heavy ions with energy of several $\mathrm{MeV}$ per nucleon deposit energy in solids mainly through the interaction with target electrons. Since the collisions with nuclei at these ion energies are improbable, the ions travel through the solid in straight paths and produce cylindrical tracks of electronic excitations. This cylindrical geometry of the track is in many amorphous materials responsible for an anisotropic plastic deformation, called ion hammering, the solid is shortened along the direction of the ion beam and lengthened normally to it [8-10]. This effect was up to now not observed in coarse grained crystalline materials. Grain boundaries are highly disordered regions. To explain the observations in nanocrystalline matter the microscopic picture of ion hammering is tentatively applied to grain boundaries in nanocrystals.

\section{Materials and Methods}

In order to study the behavior of nanocrystalline materials various layers with thickness between $500 \mathrm{~nm}$ and $7 \mu \mathrm{m}$ were prepared using physical vapor deposition (PVD). By changing the deposition parameters, it is possible to vary the grain size, which in our case was between 50 and $100 \mathrm{~nm}$. We studied pure Ti and Pd layers, and ceramic TiN layers deposited on commercially available Si (100) wafers, as well as a rolled $\mathrm{Ti}$ sheet and a monocrystalline $\mathrm{NiO}$ sample. Not knowing the origins of the observed transformation, we selected the materials with different properties in order to exclude the other possible mechanisms.

Samples were irradiated with $350 \mathrm{MeV} \mathrm{Au}$ ions at the Ionen-Strahl-Labor (ISL) at Hahn-Meitner-Institute in Berlin. Since surface energy influences texture formation, the incident angle of the ion beam was chosen to be different from the surface normal. By doing so, it is possible to separate surface effects, which would have the surface normal as preferred axis, from the influence of the ion beam, which would have the beam direction as the preferred axis. The direction of the ion beam in most experiments was chosen to be $30^{\circ}$ or $45^{\circ}$ to the surface normal.

The samples were irradiated in several sessions, and after each irradiation step the orientation of the crystalline grains was measured using Bragg diffraction of X-rays. The maximal cumulated fluence was $4 \times 10^{15}$ ions $/ \mathrm{cm}^{2}$ for $\omega$ Ti samples. The texture measurement was performed at the diffractometry experimental station at KMC2 bending magnet beamline at BESSY synchrotron light source in Berlin. The diffractometer (Huber) at the KMC2 beamline is equipped with a position sensitive detector (Bruker AXS). This detector allowed to acquire several Bragg reflections at once, including the background scattering between the reflections. Plot of the measured intensity against the sample orientation is called pole figure [11]. After necessary corrections the intensities of pole density maxima are proportional to the volume amount of grains oriented in specific direction. To acquire the complete orientation information (texture) several pole figures of non-parallel planes must be measured. The peak shape can be extracted from the measured data providing information on grain size. Estimations of the grain size and stress were, however, limited through the spatial resolution of the detector.

To measure the plastic shear deformation of the layer, the translation of the layer surface was measured in-situ during the irradiation $[12,13]$. A thin Au layer was deposited on the surface of the layer through a quadratic mesh with $60 \mu \mathrm{m}$ mesh size. During irradiation a part of the sample was covered with a copper shield. Relative translation of the surface in the irradiated surface with respect to the shaded part was measured using an optical microscope.

The effect of grain rotation was first discovered in $\alpha$-Ti, which is the room-temperature, close packed hexagonal equilibrium phase of Ti. Under high pressure Ti transforms into a structurally complex hexagonal $\omega$ phase, which is meta-stable at room temperature and atmospheric pressure. This phase transforms back to $\alpha$-Ti if tempered around $400 \mathrm{~K}$. Under certain conditions the $\omega$ phase also forms after irradiation with swift heavy ions [14]. Since $\alpha$ - $\omega$-transformation is a martensitic, it is connected with shearing of the crystalline lattice in specific directions. This lattice shearing could be the origin of the sharing of the layer and the rotation of the grains. Therefore, we studied two groups of $\mathrm{Ti}$ samples. One group was maintained in the $\alpha$ phase during irradiation, whereas the second group was irradiated under such conditions that it transformed completely in the $\omega$ phase after $10^{13}$ ions $/ \mathrm{cm}^{2}$ and stayed in this state until the end of the experiment.

Since both studied pure Ti phases were hexagonal, it was necessary to proof that the measured effects are not due to the anisotropy of the thermal dilatation. Thermal dilatation of materials with cubic crystalline structure is isotropic, and in this case would not show any effect. Cubic TiN layers were used to test this assumption.

Irradiation of the Ti layers was performed at room temperature and at liquid nitrogen temperature to study the influence of thermal effects. There was no significant difference between these measurements.

Single-crystalline $\mathrm{NiO}$ was irradiated under similar conditions. Two single crystals with orientations (111) and (001) with respect to the surface were irradiated with $\mathrm{Au}$ ions under an incidence angle of $45^{\circ}$. Due to the defects produced by the ion irradiation, the irradiated volume of the single crystal broke into a nanocrystalline material with mosaic texture, so it can be regarded as a nanocrystalline layer. Since the ion penetration depth is smaller than the sample thickness, the ions were implanted in this sample. The ion energy loss is not constant through the nanocrystallized layer, so the analogy to deposited layers is only qualitative. 
For a comparison with nanocrystalline layers, a coarsegrained Ti sheet with a grain size of $5 \mu \mathrm{m}$ was studied. $\mathrm{Pd}$ nanocrystalline material was also irradiated, since it is known that the $\mathrm{Pd}$ does not show superplastical behavior.

\section{Experimental results}

Before the irradiation the texture of all deposited samples showed a cylindrical symmetry, which is referred to as a fiber texture. The axis of symmetry is normal to the layer surface. This texture occurs in most deposited layers under influence of surface energy and interface energy to the substrate. Two different orientations of $\mathrm{NiO}$ were studied, (100) plane parallel to the sample surface and (111) plane parallel to the surface. Both Ti phases, TiN, and singlecrystalline $\mathrm{NiO}$ showed drastic changes in the texture after irradiation. Nanocrystalline Pd layers and micro-crystalline Ti sheets did not show any change up to very high fluences $\left(1 \times 10^{15}\right.$ ions $\left./ \mathrm{cm}^{2}\right)$.

The changes can be qualitatively divided in two major groups:

- The most obvious effect was the rotation of the complete orientation distribution function away from the direction of the impinging ion beam.

- The original cylindrical symmetry in the texture is disturbed, resulting in a mosaic texture, i.e. all grains have narrow angular distribution around one preferred orientation.

$\omega$-Ti and TiN showed simultaneously both effects. NiO, since it already had a mosaic texture at the beginning of the irradiation, did not show any reorientation. The texture of $\alpha$-Ti showed only the breaking of the cylindrical symmetry.

In case of $\omega$-Ti, the complete layer was transformed into the new phase after irradiation of $\alpha$-Ti with $1 \times 10^{14}$ ions $/ \mathrm{cm}^{2} \omega$-Ti layers were irradiated to the highest fluence, up to $4 \times 10^{15}$ ions $/ \mathrm{cm}^{2}$ [15]. At this point the, cylindrical symmetry was already partially destroyed. The texture of the $\omega$-phase continued to rotate until it slowed down and finally stopped at $3 \times 10^{15}$ ions $/ \mathrm{cm}^{2}$. Since the collective grain rotation can be connected with the macroscopic shearing of the layers, it can be expected that the surface of the layer translates with the rate proportional to the fluence. A thin gold layer was deposited through a mesh on the top of the Ti layer as a surface marker. After irradiating only one half of the layer, the translation of the surface could be measured. Fig. 2 shows the optical microscopy after $1.5 \times 10^{15}$ ions $/ \mathrm{cm}^{2}$.

In TiN the cylindrical symmetry was destroyed already after $2 \times 10^{14}$ ions $/ \mathrm{cm}^{2}$ (Fig. 1 ), and the rotation continued after this point. The reversibility of the rotation is verified by rotating the sample $180^{\circ}$ around the surface normal and irradiating it with the same fluence. The (002) maximum in the right part of Fig. 1 not only returned to the origin, it passed to the other side and rotated for further $20^{\circ}$. This shows that there is a transient time at the beginning of the irradiation where no rotation takes place.

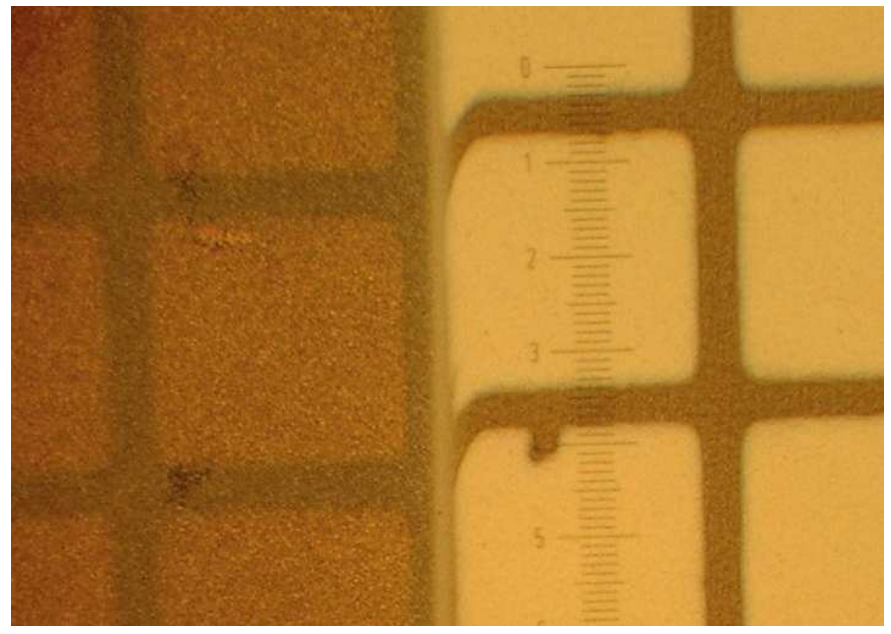

Fig. 2. Optical micrograph of the gold marker at the surface of irradiated sample. During the irradiation the left side of the sample was covered with a shield. The right side was irradiated with $1.5 \times 10^{15}$ ions $/ \mathrm{cm}^{2}$. Ion direction was $45^{\circ}$ to the surface normal. The distortion of the surface marker is used as a measure for the anisotropic dilatation of the material due to the ion irradiation.
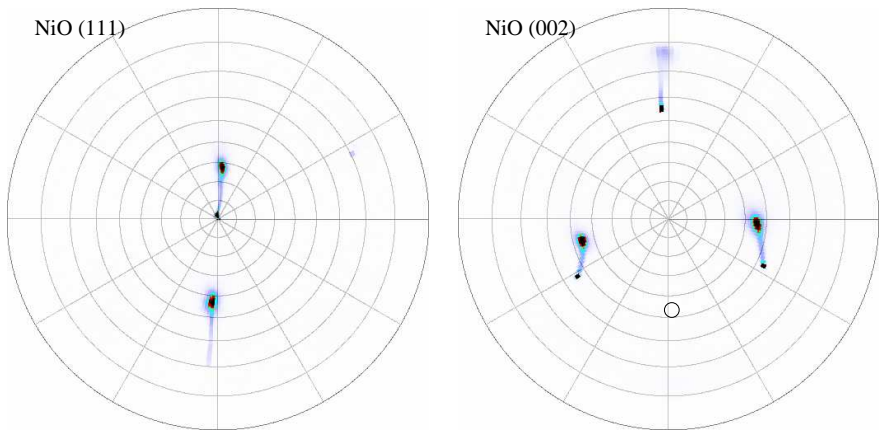

Fig. 3. (Color online) Pole figures of (111) NiO crystal after irradiation. Since the information depth for x-rays is larger than the modified layer, the original peaks of unmodified bulk material are still visible. The color scale was modified to emphasize the texture of the surface layer. During the irradiation the texture of the surface layer rotated away from the ion beam. The direction of the ion beam is marked with a circle in the figure on the right.

During the irradiation of a $\mathrm{NiO}$ single crystal, the surface layer of the monocrystalline sample is broken into nanocrystals due to the accumulation of defects. During the irradiation, these newly created nano-grains started to behave in the same way as in $\omega$-Ti and TiN, i.e they started to rotate in the direction away from the ion beam (Fig. 3). After the irradiation with $1.6 \times 10^{14}$ ions $/ \mathrm{cm}^{2}$ the texture of the sample rotated about $27^{\circ}$.

The texture in $\alpha$-Ti did not rotate in a same way as in other samples. The change in texture reflected the reorientation of the crystalline grains with respect to the direction of the ion beam. Grains in $\alpha$-Ti changed the orientation in order to bring the low-indexed planes normal to the ion beam direction. The surface normal has very small influence on the final texture. Pole figures of an $\alpha$-Ti layer irradiated from the direction $30^{\circ}$ to the surface normal are shown in Fig. 4. The result is a cylindrically symmetrical texture with the ion beam direction as the symmetry axis. 

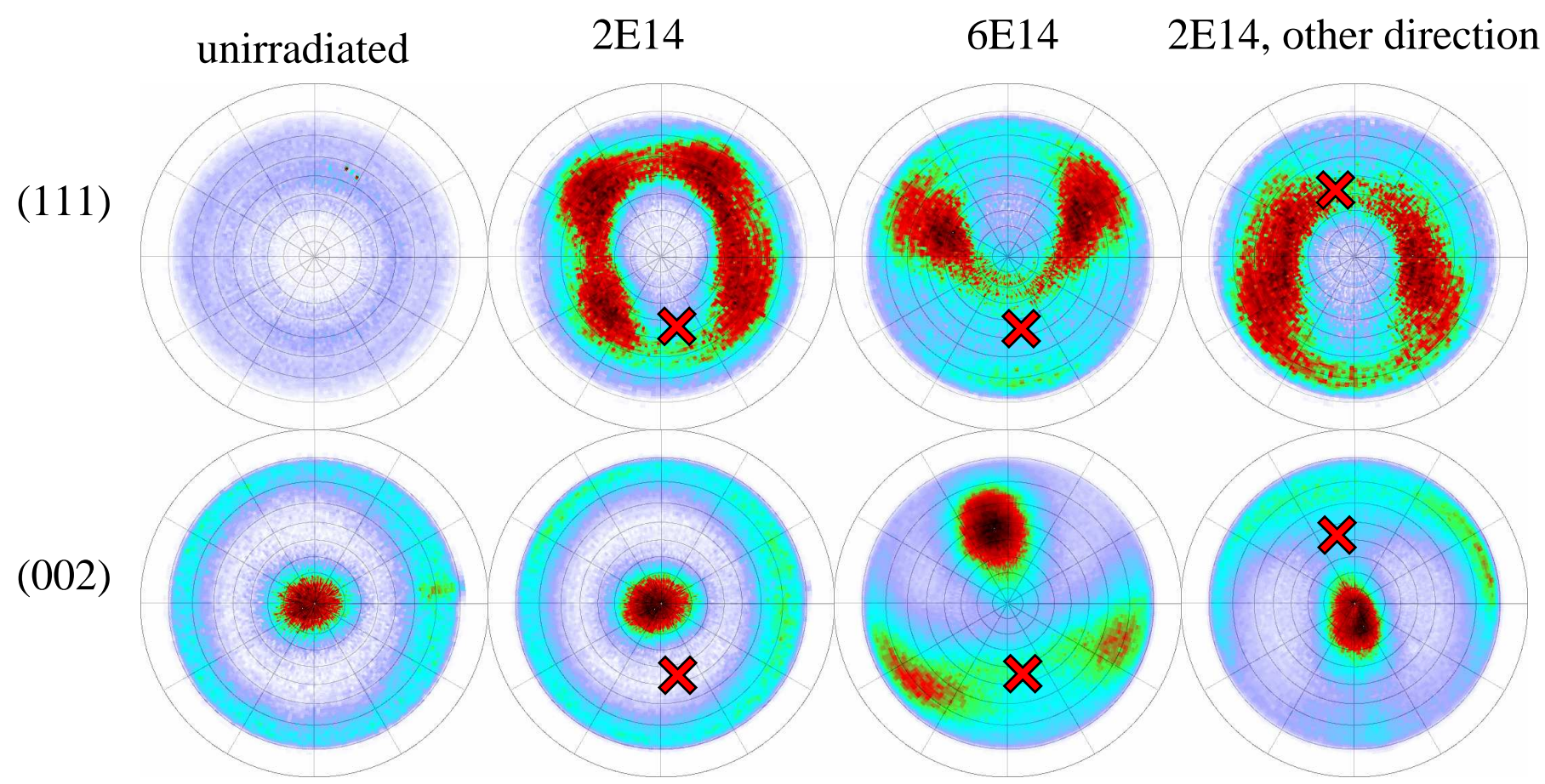

Fig. 1. (Color online) (111) and (002) pole figures in the nanocrystalline TiN layer after irradiation to different fluences (White for low intensity, blue, green, red, and black for high intensity). Fluences in ions $/ \mathrm{cm}^{2}$ are shown above the pole figures. A red 'X' marks the direction of the incident ion beam. Concentric rings originating from cylindrical symmetry are visible in the unirradiated sample. In the second pole figure, after $2 \times 10^{14}$ ions $/ \mathrm{cm}^{2}$, the cylindrical symmetry is broken, and the texture starts to rotate upwards. The rotation angle is about $5^{\circ}$. After $6 \times 10^{14}$ ions $/ \mathrm{cm}^{2}$, the rotation angle is about $45^{\circ}$. Obviously, there is a transient time where the texture does not rotate at the beginning of irradiation. To verify this, the sample in the second column was irradiated with the same fluence in opposite direction. The position of the (002) peak moved over its original position and continued to rotate about $20^{\circ}$.

In the case of the sample in the Fig. 4 there are maxima in pole figures of both measured Bragg reflections matching the direction of the ion beam. A subset of grains is oriented such that the (111) plane is normal to the ion beam direction, the other subset is oriented with the (101) plane normal to the ion beam.

The grain size can be extracted from the data acquired for the texture measurements. The Debye formula for the estimation of the grain size leads to $30-100 \mathrm{~nm}$ for the grain size in different samples at the beginning of the irradiation, but with ongoing irradiation the grains coarsened. The grains in $\omega$-Ti were larger than the resolution of the measurement $(150 \mathrm{~nm})$ after $1 \times 10^{15}$ ions $/ \mathrm{cm}^{2}$.

\section{Discussion}

Ion-beam irradiation induced texture changes in thin films are an often reported phenomenon, especially in the case of films deposited by ion beam assisted deposition (IBAD). There, the films can be biaxially textured by using well-defined ion incidence angles [16]. In principle, the orientation of the film is determined by the minimization of the surface energy leading to closed-packed lattice planes lying parallel to the substrate surface. But this is not the only determinig factor.

The influence of mechanical stress during IBAD thin film growth is such that the ion bombardment creates compres- sive strain that saturates at a certain film thickness. In order to minimize the elastic deformation, the crystallites will orient in such a way that the lattice planes with the lowest strain energy are parallel to the film surface. This is a dynamic and evolutionary process, leading to a preferred (out-of-plane) orientation of crystallites $[16,17]$. Competitive to this mechanism is the crystallite orientation due to ion channeling, as well as due to lattice orientation dependent sputtering coefficients. Both mechanisms are, again, dynamic processes and evolutionary. For a theoretical evaluation see for example $[16,17]$.

The mechanisms in swift heavy ion irradiation and IBAD are quite different: While in the first case, the dominating energy loss mechanism is the electronic energy loss, in the latter case the process is dominated by nuclear energy loss leading to high point defect densities. Therefore, the material is altered at different depths. For swift heavy ion irradiation, usually the whole thickness of the thin film is penetrated by the ions, while in ion beam assisted film growth, the penetration depth of the ions is usually only a few nanometers leaving the material underneath unharmed. While swift heavy ion irradiation is an almost instantaneous process that with increasing fluence leads to an increasing rearrangement of material that is already present, ion beam assisted growth is a dynamic process including the ion bombardment flux and the incoming flux of new film material that is to be rearranged at the film surface by 

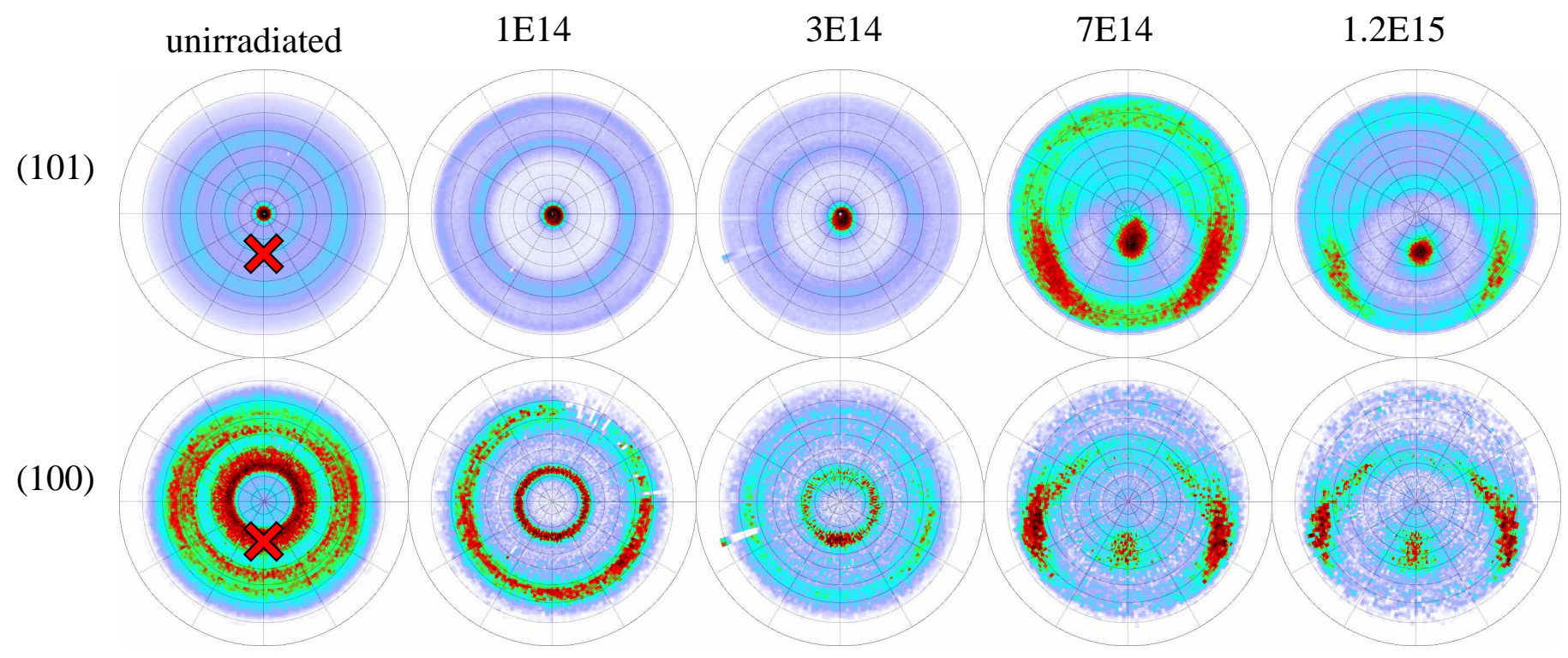

Fig. 4. (Color online) Texture rotation of the $\alpha$-Ti layer. The texture does not rotate in one direction as in other materials. Note that the texture does not discontinuously transform from the beginning to the end state. The maximum in the middle of the (101) pole figure rotates continously downwards until it reaches the direction of the ion beam, $30^{\circ}$ from its original direction.

the ion bombardment flux.

A third case of texture alteration by ion beam irradiation is the ion implantation of materials with ion energies in the medium energy range from several tens of $\mathrm{keV}$ to several $100 \mathrm{keV}$. Here, again the nuclear energy loss dominates, the altered region is of several $100 \mathrm{~nm}$ thickness. In principle, like with swift heavy ion irradiation, the material to be altered is already present. Texture changes due to medium energy ion implantation are reported: For example, Rauschenbach and Helming implanted nitrogen into thin titanium layers and they observed the formation of preferentially oriented TiN crystallites [18]. By changing the incidence angle of the nitrogen ions with respect to the sample normal, the preferred orientation of the crystallites "followed" the ion beam direction. The authors concluded that the underlying mechanism is the seed-selection by ion channeling mechanism. The influence of high compressive stress together with a slip mechanism was ruled out.

The only explanation of the results observed in the present study using swift heavy ions is that the actual grains in the solid are rotating. Since the effect is observed solely in nanocrystalline solids, we assume that the ions are either interacting with the grain boundaries, or the large amount of grain boundaries is influencing the mechanical properties of the material. The first assumption is not fulfilled at least for $\mathrm{NiO}$, since the defects which resulted in nanocrystalline material at the begin of the irradiation were created in single crystalline material.

Surface translation and the rotation of grains in the direction away from the ion beam are connected to the shearing transformation of the layer. To take a closer look to the origin of the rotation we regard for an instant a mean-field model, ignoring that we are dealing with crystalline matter. We assume that the grains are very small and very far from each other, embedded in an amorphous matrix, the grain boundary. This is correlated with the ion hammering in amorphous materials.

Ion hammering is the effect of the unisotropic dilatation of amorphous materials during the irradiation with swift heavy ions [19-22]. However, if the amorphous material is fixed on a substrate and irradiated from a direction which is not normal to the surface, it can not expand freely in the direction normal to the ion beam. The only degree of freedom is the shearing of the layer. Amorphous material translates in the direction parallel to the surface with a depth dependent velocity. This means that for a small grain embedded in this matrix, the material above the grain moves faster than the material below the grain leading to a rotational momentum on grains with the axis parallel to the surface and normal to the ion beam direction. The rotation does not depend on the grain size, so it is possible that all the grains rotate collectivly. A more quantitative discussion is given in [15].

This model describes only the mean rotation of the grains, not the other observed effects. Here we assume that the grains are not interacting, i.e., they do not collide with each other during the rotation. The slowing down of the rotation could be explained by vanishing of grain boundaries as the grains coalesce, but it does not explain the breakdown of cylindrical symmetry or why the rotation stops in highly symmetrical orientation. The processes in $\alpha$-Ti must have their origin in the crystalline matter itself. Apparently, since the effects are not observed in coarsegrained matter, the amount of the grain boundary or small size of the grains are very important for these processes.

Microscopically, ion hammering works by changing the position of many atoms by very small amounts [21]. During this short time the material is expanding normally to the 

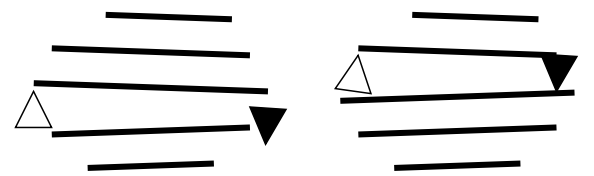

c

Fig. 5. A disclination pair moving along the grain boundary. As the consequence the complete grain is rotated.

ion direction and the atoms move away from the center of the ion track. After some time the material cools down and atoms are trapped in positions with locally minimal energy. As the thermal dilatation vanishes, the atoms are still on their new positions producing local stress.

In a crystal minimum energy atom positions are very well defined, so that with a high probability the atoms fall back into their lattice positions. In highly defective or amorphous material, there is a multitude of local minima with similar energy, so the atoms do not have to fall back into their old position. As a consequence of these small changes stress builds up. This could also happen in thin grain boundaries if the excitation energy is sufficiently large.

Grain boundaries are disordered thin layers separating neighboring crystalline grains. During the ion bombardment these thin layers are sheared, so that the neighboring grains slide with respect to each other. The net effect of the shearing of all grain boundaries is macroscopic dilatation in the direction normal to the beam and shortening in the direction of the ion beam. This is equivalent to the macroscopically measured ion hammering in amorphous materials, and will lead to translation of the surface and rotation of the grains. The grain boundaries do not even have to be amorphous, there are other possible mechanisms which can contribute to the shearing between grains. One model originates from standard plasticity theory. The prerequisite for shearing is the existence of lattice defects with a high mobility along the grain boundaries. Due to the small size of the grains, dislocation loops move outside of the grain and pile up at the grain boundary. Ovid'ko describes such a process during the superplastic deformation of the nanocrystalline materials [23-26]. A disclination dipole is created when the pile-up stress at the grain boundary triple lines becomes too large. Moving of the disclination dipole along the grain boundary can be responsible for shearing of the layer, as well as the rotation of the grains (Fig. 5).

Additionally, this process allows for the rotation of the grains in other directions, because the grain boundaries are not all parallel. In a homogeneous solid the only component of the rotation is the one around the axis lying in the sample surface and normal to the ion beam. Even if the momentum acting on the grains is in the same direction, due to the crystalline structure grains have axes of "easy rotation". Since the disclination energy and mobility depend on crystalline structure, the axis of grain rotation depends on its crystalline structure. This could explain the breaking of the cylindrical symmetry, since the grains are going to rotate around other axes. Moreover, this effect could be responsible for the slowdown of the rotation and the symmetry of the final state.

The disclination model of ion hammering in nanocrystalline solids is still very descriptive. To make it more quantitative, more experimental data is needed, as well as more theoretical understanding. An estimate of the orientation distribution of the grain boundaries could be gained from highly resolved electron microscopy. Unfortunately, very little is known about the elastic properties of $\omega$-Ti, which is the experimentally best studied case.

\section{Acknowledgments}

We would like to thank Dagmar Frischke and Axel Wenzel for their careful preparation of the titanium layers and Hans-Dieter Mieskes for stimulating discussions.

\section{References}

[1] H. Gleiter, Acta Mater. 48 (2000) 1

[2] D. Wolf, V. Yamakov, S. R. Phillpot, A. Mukherjee, H. Gleiter, Acta Mater. 53 (2005) 1.

[3] I. Szlufarska, A. Nakano, P. Vashista, Science 309 (2005) 911.

[4] A. Subramaniam, C. T. Koch, R. M. Cannon, M. Ruhle, Mat. Sci. Eng. A422 (2006) 3.

[5] C. Langlois, M. Hytch, S. Lartigue-Korinek, Y. Champion, Metal. Mat. Trans. A36 (2005) 3451.

[6] P. M. Derlet, H. van Swygenhoven, Phys. Rev. B 67 (2003) 014202 .

[7] S. von Alfthan, P. D. Haynes, K. Kaski, A. P. Sutton, Phys. Rev. Lett 96 (2006) 055505.

[8] H. Trinkaus, A. I. Ryazanov, Phys. Rev. Lett. 74 (25) (1995) $5072-75$.

[9] H. Trinkaus, Nucl. Instr. Meth. B107 (1995) 155.

[10] H. Trinkaus, Nucl. Instr. Meth. B146 (1998) 204.

[11] B. D. Cullity, Elements of X-ray diffraction, Addison-Wessley series in metalurgy, Addison-Wessley Publishing Company, Inc., Ch. 9.6., p. 272 .

[12] S. Klaumünzer, Mater.Sci. For. 97-99 (1992) 623-630.

[13] A. Hedler, S. L. Klaumünzer, W. Wesch, Nature Materials 3 (2004) 804.

[14] H. Dammak, A. Dunlop, D. Lesueur, Nucl. Instr. Meth. B107 (1996) 204-211.

[15] I. Zizak, N. Darowski, S. Klaumünzer, G. Schumacher, J. W. Gerlach, W. Assmann, Phys. Rev. Lett., accepted for publication.

[16] B. Rauschenbach, J. W. Gerlach, Cryst. Res. Technol. 35 (2000) $675-688$.

[17] G. Carter, Phys. Rev. B 62 (2000) 8376.

[18] B. Rauschenbach, K. Helmig, Nucl.Instr. Meth. in Phys. Res. B42 (1989) 216.

[19] A. Gutzmann, S. Klaumünzer, P. Meier, Phys. Rev. Lett. 74 (1995) 2256.

[20] A. Gutzmann, S. Klaumünzer, Nucl. Instr. Meth. B127/128 (1997) 12.

[21] M. D. Hou, S. Klaumünzer, G. Schumacher, Phys. Rev. B41 (1990) 1144.

[22] S. Klaumünzer, Nucl. Instr. Meth. B225 (2004) 136.

[23] I. A. Ovid'ko, Science 296 (2002) 2386.

[24] I. A. Ovid'ko, Int. Mat. Rev. 50 (2005) 65.

[25] M. Y. Gutkin, I. A. Ovid'ko, N. V. Skiba, Acta Mat. 51 (2003) 4059.

[26] M. Y. Gutkin, I. A. Ovid'ko, Appl. Phys. Lett. 87 (2005) 251916. 